\title{
Amyloid and tau signatures of brain metabolic decline in preclinical Alzheimer's disease
}

\author{
Tharick A. Pascoal ${ }^{1}$. Sulantha Mathotaarachchi ${ }^{1} \cdot$ Monica Shin $^{1}$. Ah Yeon Park ${ }^{2}$. Sara Mohades ${ }^{1}$. \\ Andrea L. Benedet ${ }^{1}$ - Min Su Kang ${ }^{1} \cdot$ Gassan Massarweh $^{3}$ - Jean-Paul Soucy ${ }^{3,4}$ - Serge Gauthier ${ }^{5}$. \\ Pedro Rosa-Neto ${ }^{1,3,5,6}$. for the Alzheimer's Disease Neuroimaging Initiative
}

Received: 12 September 2017 / Accepted: 2 January 2018 / Published online: 2 February 2018

(C) The Author(s) 2018. This article is an open access publication

\begin{abstract}
Purpose We aimed to determine the amyloid $(\mathrm{A} \beta)$ and tau biomarker levels associated with imminent Alzheimer's disease (AD) - related metabolic decline in cognitively normal individuals.

Methods A threshold analysis was performed in 120 cognitively normal elderly individuals by modelling 2-year declines in brain glucose metabolism measured with $\left[{ }^{18} \mathrm{~F}\right]$ fluorodeoxyglucose $\left(\left[{ }^{18} \mathrm{~F}\right] \mathrm{FDG}\right)$ as a function of $\left[{ }^{18} \mathrm{~F}\right]$ florbetapir $\mathrm{A} \beta$ positron emission tomography (PET) and cerebrospinal fluid phosphorylated tau biomarker thresholds. Additionally, using a novel voxel-wise analytical framework, we determined the sample sizes needed to test an estimated $25 \%$ drugeffect with $80 \%$ of power on changes in FDG uptake over 2 years at every brain voxel.

Results The combination of $\left[{ }^{18} \mathrm{~F}\right]$ florbetapir standardized uptake value ratios and phosphorylated-tau levels more than one standard deviation higher than their respective thresholds for biomarker abnormality was the best predictor of metabolic decline in individuals with preclinical AD. We also found that a clinical trial using these thresholds would require as few as 100 individuals to test a $25 \%$ drug effect on AD-related metabolic decline over 2 years.

Conclusions These results highlight the new concept that combined $\mathrm{A} \beta$ and tau thresholds can predict imminent neurodegeneration as an alternative framework with a high statistical power for testing the effect of disease-modifying therapies on $\left[{ }^{18} \mathrm{~F}\right] \mathrm{FDG}$ uptake decline over a typical 2-year clinical trial period in individuals with preclinical AD.
\end{abstract}

Keywords Amyloid-PET $\cdot\left[{ }^{18} \mathrm{~F}\right] \mathrm{FDG}$ PET $\cdot$ Preclinical Alzheimer's disease $\cdot$ Phosphorylated tau

Data used in preparation of this article were obtained from the Alzheimer's Disease Neuroimaging Initiative (ADNI) database (adni. loni.usc.edu). As such, the investigators within the ADNI contributed to the design and implementation of ADNI and/or provided data but did not participate in analysis or writing of this report. A complete listing of ADNI investigators can be found at: http://adni.loni.usc.edu/wp-content/ uploads/how_to_apply/ADNI_Acknowledgement_List.pdf

Electronic supplementary material The online version of this article (https://doi.org/10.1007/s00259-018-3933-3) contains supplementary material, which is available to authorized users.

Pedro Rosa-Neto

pedro.rosa@mcgill.ca

1 Translational Neuroimaging Laboratory, The McGill University Research Centre for Studies in Aging, Douglas Hospital, McGill University, 6875 La Salle Blvd - FBC room 3149, Montreal, QC H4H 1R3, Canada

2 Statistical Laboratory, University of Cambridge, Cambridge, UK
3 Montreal Neurological Institute, Montreal, Canada

4 PERFORM Centre, Concordia University, Montreal, Canada

5 Alzheimer's Disease Research Unit, The McGill University Research Centre for Studies in Aging, Douglas Hospital, McGill University, Montreal, Canada

6 Department of Neurology and Neurosurgery, McGill University, Montreal, Canada 


\section{Introduction}

The preclinical stages of Alzheimer's disease (AD) have become the main focus of therapeutic clinical trials given the assumption that better outcomes can be achieved with changes in the course of the disease before the appearance of cognitive symptoms $[1,2]$. The International Working Group and the American Alzheimer's Association have recently characterized preclinical $A D$ as the presence of amyloid- $\beta(A \beta)$ and tau abnormalities in cognitively normal persons [1]. Although these individuals show greater rates of disease progression than cognitively normal biomarker-negative individuals, most of them remain stable over typical clinical trial periods $[1,3]$. Therefore, a critical next step proposed by the working groups was to identify, from among individuals with preclinical AD, those with the highest likelihood of disease progression within time frames acceptable for clinical trial designs taking into account financial, medical, and ethical considerations [1].

Its slow rate of change makes the use of cognition as the primary outcome of clinical trials in individuals with preclinical AD difficult because it leads to prohibitively high sample sizes and long follow-up times [4]. Hence, surrogate measurements of disease progression using established biomarkers of neurodegeneration might provide a useful alternative for such trials [5]. In fact, few studies have tested changes in structural magnetic resonance imaging (MRI) as a possible surrogate marker for preclinical AD clinical trials. For example, a recent study by the Alzheimer's Disease Neuroimaging Initiative (ADNI) has suggested that to test for structural changes on MRI in a population of individuals with preclinical $A D$ enriched on the basis of $A \beta$ and tau biomarker positivity, sample size estimates are prohibitively large [3]. In this regard, changes in $\left[{ }^{18} \mathrm{~F}\right]$ fluorodeoxyglucose $\left(\left[{ }^{18} \mathrm{~F}\right] \mathrm{FDG}\right)$ uptake have been suggested for following disease progression and monitoring therapeutic effects [6-11]. Indeed, $\left[{ }^{18} \mathrm{~F}\right] \mathrm{FDG}$ positron emission tomography (PET) is one of the most important biomarkers of $\mathrm{AD}$ with applications in the research and clinical settings. However, the characteristics of $\left[{ }^{18} \mathrm{~F}\right] \mathrm{FDG}$ uptake as a surrogate marker of preclinical AD in clinical trials are not fully known. Indeed, the inclusion of cognitively normal individuals harbouring $A \beta$ and tau, and with abnormal $\left[{ }^{18} \mathrm{~F}\right] \mathrm{FDG}$ uptake could be argued as an interesting strategy for these trials, since such individuals have a higher probability of developing further neurodegeneration and cognitive symptoms [1]. However, it is reasonable to suggest that the absence of baseline neurodegeneration might offer a more favourable pathophysiological scenario for preventive therapies aiming to mitigate disease progression [2].

Recent literature indicates that $A \beta$ and tau pathologies may arise independently and, at some pathophysiological point, synergistically potentiate imminent neurodegeneration in preclinical $\mathrm{AD}[2,12-14]$. Notably, this framework infers the existence of thresholds for $A \beta$ and tau pathologies associated with the triggering of their deleterious synergy in potentiating neurodegeneration. However, the fact that the majority of $A \beta$-positive plus tau-positive individuals with preclinical AD remain pathophysiologically stable over long periods suggests that these thresholds are greater than their respective individual thresholds for abnormality [1]. Thus, determination of the $A \beta$ and tau biomarker thresholds associated with imminent neurodegeneration might provide complementary information in individuals with preclinical $\mathrm{AD}$ destined to develop AD-related progression.

In this study, we tested the hypothesis that the combination of optimized $A \beta$ and tau thresholds predictive of neurodegeneration might provide an alternative framework with a high statistical power for testing the efficacy of the emerging disease-modifying therapies. This framework has the potential to select preclinical individuals on the verge of AD-related progression, considering metabolic changes as indices of neurological decline.

\section{Materials and methods}

\section{Participants}

Data used in this study were obtained from the ADNI database (adni.loni.usc.edu). The ADNI was launched in 2003 as a public-private partnership led by the Principal Investigator Michael W. Weiner. The primary goal of ADNI has been to test whether serial MRI, PET, other biological markers, and clinical and neuropsychological assessment can be combined to measure the progression of mild cognitive impairment and early AD. For the present analysis, we selected 120 cognitively normal ADNI participants in whom cerebrospinal fluid (CSF) phosphorylated-tau (p-tau) was determined and $\left[{ }^{18} \mathrm{~F}\right]$ florbetapir PET was performed at the same baseline visit, and $\left[{ }^{18} \mathrm{~F}\right] \mathrm{FDG}$ PET at the baseline visit and at the 2-year follow-up visit.

\section{CSF analysis}

CSF p-tau and CSF A $\beta$ were quantified with the INNOBIA AlzBio3 immunoassay using the multiplex xMAP Luminex platform (Luminex Corp, Austin, TX). The CSF data used in the analysis were selected from the ADNI files "UPENNBIOMK5-8.csv", and all the values for each subject were obtained from the same file. The standard published ADNI threshold for p-tau abnormality of $>23 \mathrm{pg} / \mathrm{ml}$ was used in this analysis $[15,16]$. Further details of methods for CSF acquisition and quantification can be found at www.adni-info.org. 


\section{MRI/PET}

MRI and PET acquisitions followed the ADNI protocols (http://adni.loni.usc.edu/methods). The MRI T1-weighted images underwent nonuniformity correction, brain masking and segmentation using the Brain Extraction based on nonlocal Segmentation Technique [17]. The T1-weighted images were then processed using the CIVET image-processing pipeline and registered using a nine-parameter affine transformation and nonlinearly spatially normalized to the MNI 152 template [18]. PET images were smoothed using a volumetric gaussian kernel with a full-width at half-maximum of $8 \mathrm{~mm}$. Subsequently, linear registration and nonlinear normalization to the MNI 152 template were performed with the linear and nonlinear transformation derived from the automatic PET to MRI transformation and the individual's anatomical MRI coregistration. $\left[{ }^{18} \mathrm{~F}\right]$ Florbetapir and $\left[{ }^{18} \mathrm{~F}\right] \mathrm{FDG}$ standardized uptake value ratio (SUVR) maps were generated using the cerebellum grey matter and the pons as reference regions, respectively [13]. Global PET SUVR values for each subject were estimated from the precuneus, prefrontal, orbitofrontal, parietal, temporal, and cingulate cortices. In our pipeline, $30 \%$ of controls were $\mathrm{A} \beta$-positive using a standard $\left[{ }^{18} \mathrm{~F}\right]$ florbetapir SUVR threshold of 1.15, which is consistent with ADNI publications $[19,20]$. Further details regarding our imageprocessing pipeline can be found elsewhere [13, 14].

\section{Statistical methods}

\section{Threshold analysis}

We determined the thresholds predictive of metabolic decline by modelling $\Delta\left[{ }^{18} \mathrm{~F}\right] \mathrm{FDG}$ (( $\left.\frac{\text { SUVR Follow up-SUVR Baseline }}{\text { SUVR Baseline }}\right) *$ $\left.\frac{100}{\Delta \text { Time }}\right)$ as a function of baseline $\left[{ }^{18} \mathrm{~F}\right]$ florbetapir and $\mathrm{p}$-tau thresholds in regions of interest. The regions were segmented using the coordinates in the MNI ICBM atlas [21], and the threshold analysis was performed using GraphPad Prism 6.0 software. $R$-squared and the $F$-test were used to evaluate the goodness-of-fit, while the Akaike information criterion (AIC) was used to compare linear and nonlinear functions. Nonlinear associations were assumed to be sigmoidal, as discussed previously [22], and were formulated as follows:

$Y=a+\left(\frac{b}{1+\mathrm{e}^{\frac{-(X-c)}{d}}}\right)$

In this equation, $Y$ is the $\Delta\left[{ }^{18} \mathrm{~F}\right] \mathrm{FDG}, X$ is the biomarker threshold, and $a, b, c$ and $d$ are the parameters of the fitting, where $a$ corresponds to the lower asymptote of the $\Delta\left[{ }^{18} \mathrm{~F}\right] \mathrm{FDG}$ at the unit of $Y, b$ corresponds to the total change in $\Delta\left[{ }^{18} \mathrm{~F}\right] \mathrm{FDG}$ as a function of biomarkers (value between the lower and the upper asymptote) at the unit of $Y, c$ is the $\Delta\left[{ }^{18} \mathrm{~F}\right] \mathrm{FDG}$ at the inflection point of the curve at the unit of $X$, and $d$ represents the curve steepness. The biomarker threshold for imminent metabolic decline was defined as the value where the curvatures changed sign $(X(c))$. This point was assumed as the magnitude that needs to be reached by the biomarker for the determination of a significant 2-year metabolic decline.

\section{Group comparison analysis}

Groups were compared using analysis of covariance (ANCOVA) using the R statistical software package version 3.1 to test for significant differences in metabolic decline between biomarker groups and also in demographic differences between biomarker groups for continuous variables, whereas the chi-squared test was used for the categorical ones. The $P$ values are presented after correction for multiple comparisons testing using Bonferroni correction at a significance level of 0.05 , and differences in metabolic decline among the biomarker groups were further adjusted for age, gender, education, and $A P O E-\varepsilon 4$ carrier status.

\section{Voxel-wise sample size calculation}

A voxel-wise power analysis was performed using MATLAB 15 a software with a novel analytical tool adapted to assess the sample size necessary for a clinical trial testing for a drug effect on $\Delta\left[{ }^{18} \mathrm{~F}\right]$ FDG uptake at every brain voxel [23]. To the best of our knowledge, this is the first PET study in which voxel-wise power calculations have been performed. The sample calculations estimated the number of subjects required to detect $25 \%$ slowing in $\Delta\left[{ }^{18} \mathrm{~F}\right] \mathrm{FDG}$ uptake for a hypothetical disease-modifying therapy versus placebo with $80 \%$ of power at a $5 \%$ level $[24,25]$. The analysis was performed with a well-described formula across the biomarker groups [24-27]:

sample size $(\operatorname{voxel}(\mathrm{x}, \mathrm{y}, \mathrm{z}))=(\partial+\delta)^{2} * \frac{2 \sigma^{2}}{\left(\Delta \mu^{*} \beta\right)^{2}}$

We used $\partial=0.842$ to power at $80 \%$ and $\delta=1.96$ to test a significance of $5 \% ; \Delta \mu$ is the average percentage of change in the signal intensity of $\left[{ }^{18} \mathrm{~F}\right] \mathrm{FDG} ; \beta$ is the drug effect of 0.25 to reflect the difference in $\left[{ }^{18} \mathrm{~F}\right] \mathrm{FDG}$ uptake between drug and placebo groups; $\sigma$ is the standard deviation (SD) of $\left[{ }^{18} \mathrm{~F}\right] \mathrm{FDG}$ uptake changes, and $(x, y, z)$ is the coordinate of each voxel. Importantly, the parametric images showed the regions with a significant metabolic decline over 2 years after correction for multiple comparisons testing using a false discovery rate at $P<0.001$. 


\section{Results}

\section{Threshold analysis}

A sigmoidal curve was the best fit to represent the decline in $\left[{ }^{18} \mathrm{~F}\right] \mathrm{FDG}$ uptake over 2 years as a function of $\left[{ }^{18} \mathrm{~F}\right]$ florbetapir thresholds in the mediobasal temporal cortex $\left(R^{2}=0.98\right.$, AIC 11.83 with probability of correctness, PC, $96.57 \%$ versus $3.43 \%$ for the linear model), orbitofrontal cortex $\left(R^{2}=0.97\right.$, AIC 17.11, PC 99.98\%), anterior cingulate cortex $\left(R^{2}=0.97\right.$, AIC 1.86 , PC cortex $71.68 \%)$, and posterior cingulate cortex $\left(R^{2}=0.97\right.$, AIC 6.43 , PC $96.13 \%$ ). Based on the inflection point of the preferred sigmoidal model, a curve-fitting threshold analysis on averaged clusters $\left(R^{2}=0.98\right.$, AIC 5.26 , PC $93.28 \%$ ) revealed that the optimal $\left[{ }^{18} \mathrm{~F}\right]$ florbetapir threshold predictive of imminent metabolic decline was 1.228 (95\% CI 1.205-1.253, 1 SD higher than the standard threshold; Fig. 1a, b; Supplementary Table 1). Interestingly, although $\left[{ }^{18} \mathrm{~F}\right]$ florbetapir SUVR was highly

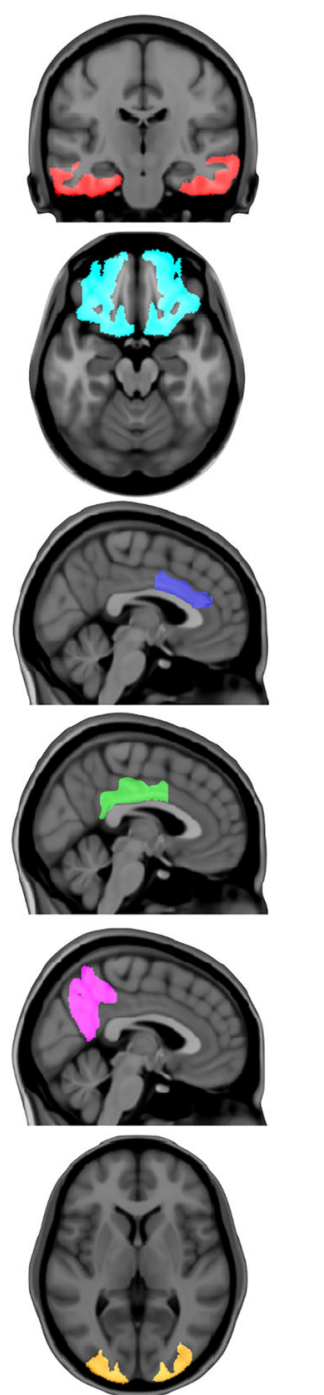

a

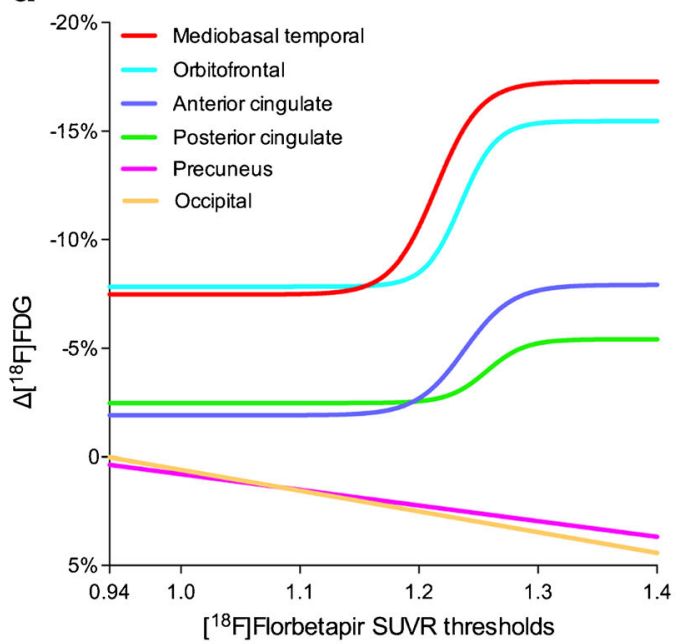

C

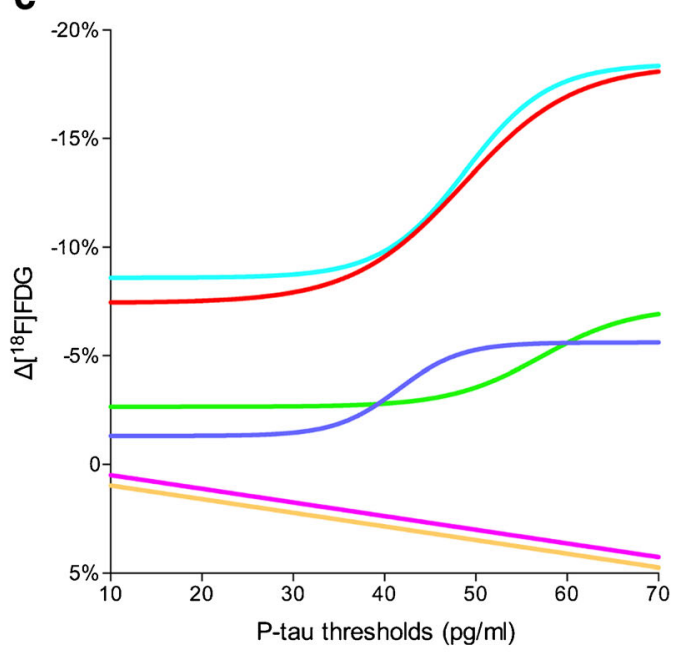

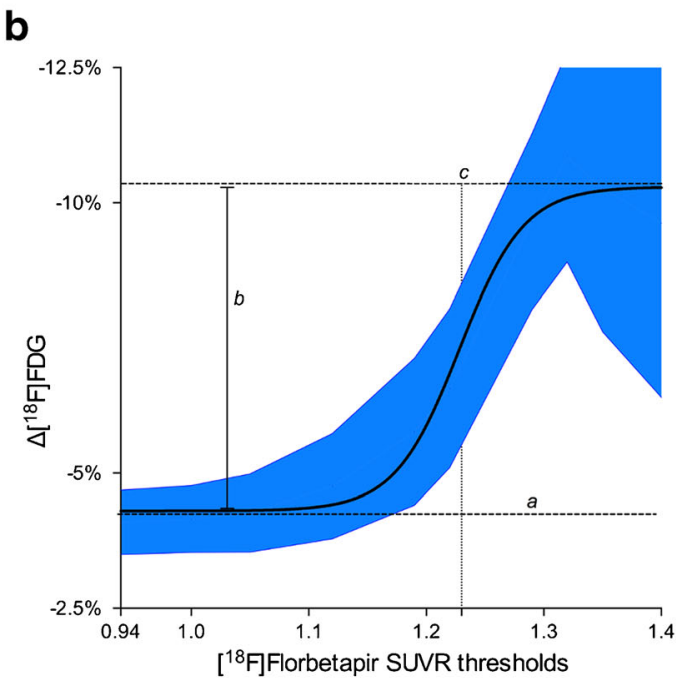

d

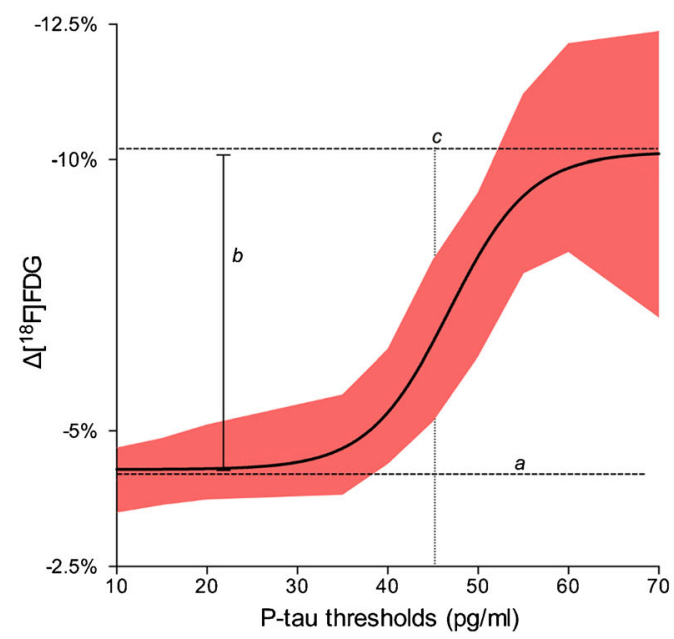

Fig. $1 \quad A \beta$ and tau thresholds associated with imminent metabolic decline are higher than their standard thresholds for biomarker abnormality. Curves represent changes in $\left[{ }^{18} \mathrm{~F}\right] \mathrm{FDG}$ uptake over 2 years within anatomically segregated clusters as a function of baseline $\left[{ }^{18} \mathrm{~F}\right]$ florbetapir SUVR and CSF p-tau thresholds. a As a function of $\left[{ }^{18} \mathrm{~F}\right]$ florbetapir thresholds, $\left[{ }^{18} \mathrm{~F}\right] \mathrm{FDG}$ uptake decline fits a sigmoidal curve in the mediobasal temporal cortex $\left(R^{2}=0.98\right)$, the orbitofrontal cortex $\left(R^{2}=0.97\right)$ the anterior cingulate cortex $\left(R^{2}=0.97\right)$ and the posterior cingulate cortex $\left(R^{2}=0.97\right)$. b In the average clusters, the inflection point of $\left[{ }^{18} \mathrm{~F}\right] \mathrm{FDG}$ uptake decline was at $\left[{ }^{18} \mathrm{~F}\right]$ florbetapir SUVR 1.228 $\left(R^{2}=0.98,95 \%\right.$ CI $\left.1.205-1.253\right)$. c As a function of CSF p-tau thresholds, $\left[{ }^{18} \mathrm{~F}\right] \mathrm{FDG}$ uptake decline fitted a sigmoidal curve in the mesiobasal temporal cortex $\left(R^{2}=0.97\right)$, the orbitofrontal cortex $\left(R^{2}=\right.$ $0.96)$, the anterior cingulate cortex $\left(R^{2}=0.98\right)$ and the posterior cingulate cortex $\left(R^{2}=0.94\right)$. $\mathbf{d}$ The inflection point of the sigmoidal curve in the averaged clusters was at a CSF p-tau of $45 \mathrm{pg} / \mathrm{ml}$ (95\% CI 43.72-47.9). Notably, clusters in the precuneus and occipital lobe did not have any linear or sigmoidal association with biomarker thresholds. In the curves, $a$ corresponds to the lower asymptote, $b$ corresponds the total change in $\Delta\left[{ }^{18} \mathrm{~F}\right] \mathrm{FDG}$ uptake, and $c$ is the $\Delta\left[{ }^{18} \mathrm{~F}\right] \mathrm{FDG}$ uptake at the inflection point of the curve 
correlated with CSF A $\beta$ (Spearman's rho $=0.68$, $P<0.0001$ ), CSF A $\beta$ thresholds did not significantly model the declines in $\left[{ }^{18} \mathrm{~F}\right] \mathrm{FDG}$ uptake in the above regions in our population.

A sigmoidal curve was the best fit to represent declines in $\left[{ }^{18} \mathrm{~F}\right] \mathrm{FDG}$ uptake as a function of p-tau thresholds in the mesiobasal temporal cortex $\left(R^{2}=0.97\right.$, AIC 7.53 , PC $97.74 \%)$, the orbitofrontal cortex $\left(R^{2}=0.96\right.$, AIC 7.88 , PC 98\%), the anterior cingulate cortex $\left(R^{2}=0.98\right.$, AIC 23 , PC $>99 \%)$, the posterior cingulate cortex $\left(R^{2}=0.94\right.$, AIC 5.15, PC >96\%), and averaged clusters $\left(R^{2}=0.94\right.$, AIC 8, PC 98.23\%). Curve-fitting threshold analysis on averaged clusters revealed an inflection point of $45 \mathrm{pg} / \mathrm{ml}$ (95\% CI 43.72-47.9 pg/ml, 1.3 SD higher than the standard threshold; Fig. 1c, d; Supplementary Table 1).

In addition, we plotted $\Delta\left[{ }^{18} \mathrm{~F}\right] \mathrm{FDG}$ uptake inside clusters in $\left[{ }^{18} \mathrm{~F}\right]$ florbetapir-positive plus p-tau-positive groups segregated using all possible combinations of thresholds for both biomarkers $(n=14,400)$. The 3D plot confirms that individuals with preclinical $\mathrm{AD}$ defined using progressively higher biomarker thresholds, with both

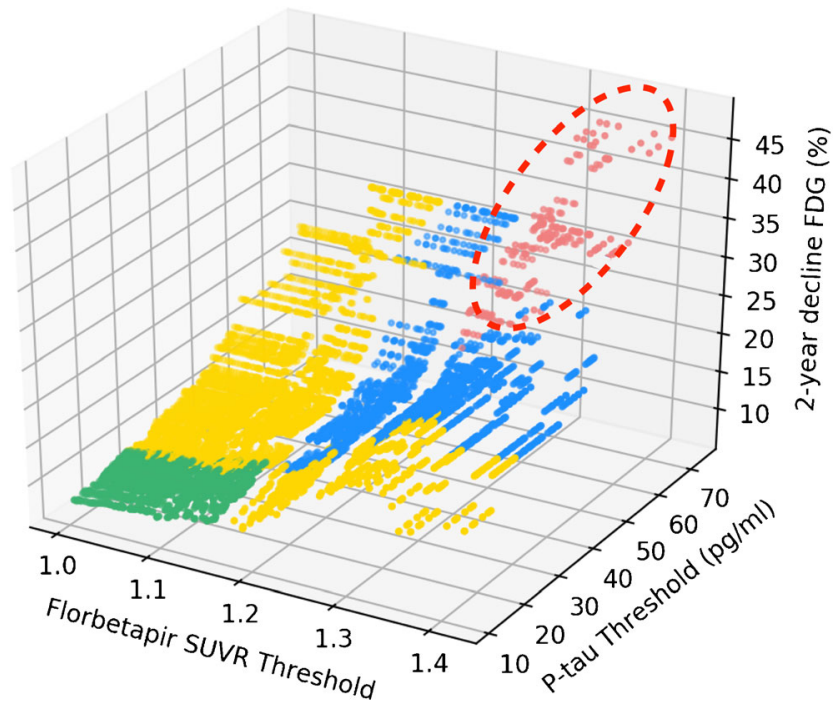

Fig. 2 Preclinical $A D$ groups with progressively higher $A \beta$ and tau thresholds, with both biomarker levels greater than those for imminent metabolic decline, had progressively higher rates of $\left[{ }^{18} \mathrm{~F}\right] \mathrm{FDG}$ hypometabolism. The dots in the 3D plot represent the mean metabolic decline in clusters over 2 years in $\mathrm{A} \beta$-positive plus p-tau-positive groups segregated using all possible combinations $(n=14,400)$ of threshold values for $\left[{ }^{18} \mathrm{~F}\right]$ florbetapir SUVR and CSF $\mathrm{p}$-tau. The groups with both biomarker thresholds lower than the standard values (green), with only one biomarker threshold higher than the standard thresholds (yellow) and with both biomarker thresholds higher than the standard values with at least one lower than the threshold (blue) for imminent metabolic decline did not show a significant 2 -year decline in $\left[{ }^{18} \mathrm{~F}\right] \mathrm{FDG}$ uptake. On the other hand, $A \beta$-positive plus p-tau-positive groups segregated using both thresholds equal to or higher than the proposed thresholds showed progressively higher rates of metabolic decline with the progressive increase in the biomarker threshold values (red) biomarker levels greater than the thresholds for imminent metabolic decline, had progressively higher rates of $\Delta\left[{ }^{18} \mathrm{~F}\right]$ FDG hypometabolism (Fig. 2).

\section{Group comparison analysis}

Applying the new criteria for preclinical AD with standard thresholds [1], out of 120 individuals, 24 (20\%) were biomarker-negative, $63(53 \%)$ were at risk of $\mathrm{AD}$ (only one biomarker abnormality), while 33 (27\%) showed preclinical $\mathrm{AD}$ with both $\mathrm{A} \beta$ and $\mathrm{p}$-tau abnormalities. Using the proposed combination of $\left[{ }^{18} \mathrm{~F}\right]$ florbetapir (SUVR $>1.228)$ and CSF $\mathrm{p}$-tau $(>45 \mathrm{pg} / \mathrm{ml})$ thresholds predictive of imminent metabolic decline, out of 33 individuals with preclinical AD, 17 (14\%) had both biomarkers above the thresholds. Importantly, voxel-wise comparison revealed that all the above biomarker groups did not show any significant differences in $\left[{ }^{18} \mathrm{~F}\right] \mathrm{FDG}$ uptake at the baseline visit. Demographics and key sample characteristics of the individuals of the population across biomarker groups are summarized in Table 1.

ANCOVA of the average decline in $\left[{ }^{18} \mathrm{~F}\right] \mathrm{FDG}$ uptake in each region confirmed that individuals with $\left[{ }^{18} \mathrm{~F}\right]$ florbetapir plus $\mathrm{p}$-tau levels above the proposed thresholds showed the highest rates of $\left[{ }^{18} \mathrm{~F}\right] \mathrm{FDG}$ decline in the mediobasal temporal, orbitofrontal, anterior cingulate and posterior cingulate cortices in our population (Fig. 3). The biomarker groups did not show any differences in average $\left[{ }^{18} \mathrm{~F}\right] \mathrm{FDG}$ uptake at baseline in any region of interest. Interestingly, in the precuneus and the occipital lobe, there were no significant differences in the decline in $\left[{ }^{18} \mathrm{~F}\right] \mathrm{FDG}$ uptake across the biomarker groups.

\section{Voxel-wise sample size calculation}

A voxel-wise power analysis - free of anatomical assumptions - further confirmed that identifying individuals with both biomarkers above the proposed thresholds is the best approach to population enrichment in therapeutic trials using changes in voxel-wise $\left[{ }^{18} \mathrm{~F}\right] \mathrm{FDG}$ uptake over 2 years in large clusters in the orbitofrontal, anterior and posterior cingulate, and mediobasal and lateral temporal cortices as a surrogate marker of preclinical $\mathrm{AD}$ requiring as few as 50 individuals per trial arm (Fig. 4).

Additionally, to ensure that the results of our voxelwise power calculation were not affected by any issues related to the voxel-wise approach, we averaged the SUVR values inside segregated regions and performed the same analyses for each region using these average SUVRs as the outcome (Supplementary Table 2). Using the average $\Delta\left[{ }^{18} \mathrm{~F}\right] \mathrm{FDG}$ uptake in the mediobasal temporal cortex as a surrogate marker, a study population 
Table 1 Demographics and key characteristics of the population in each biomarker group

\begin{tabular}{|c|c|c|c|c|c|}
\hline Characteristic & $\begin{array}{l}\text { Biomarker- } \\
\text { negative }\end{array}$ & At risk of $\mathrm{AD}$ & $\begin{array}{l}\text { Preclinical AD below } \\
\text { thresholds }\end{array}$ & $\begin{array}{l}\text { Preclinical AD above } \\
\text { thresholds }\end{array}$ & $P$ value \\
\hline No. of subjects & 24 & 63 & 16 & 17 & \\
\hline Age (years), mean (SD) & $75.1(7.4)$ & $73.9(6.1)$ & $74.9(6.2)$ & $78.6(5.1)$ & 0.37 \\
\hline Male, $n(\%)$ & $14(58)$ & $35(55)$ & $7(44)$ & $7(42)$ & 0.3 \\
\hline Education (years), mean (SD) & $17.7(2.5)$ & $16.3(2.9)$ & $15.6(2.4)$ & $16.7(2.5)$ & 0.6 \\
\hline \multicolumn{6}{|l|}{ MMSE score, mean (SD) } \\
\hline Baseline & $29.1(1.4)$ & $29.2(0.96)$ & $28.9(0.97)$ & $29(0.92)$ & 0.81 \\
\hline Follow-up & $29(1.6)$ & $29(1.1)$ & $28.4(1.9)$ & $28.6(1.3)$ & 0.27 \\
\hline$A P O E-\varepsilon 4$ carrier, $n(\%)$ & $1(4)$ & $17(27)$ & $6(38)$ & $6(35)$ & 0.06 \\
\hline P-tau (pg/ml), mean (SD) & $19.3(2.9)$ & $35.8(14.8)^{*}$ & $36.5(8.9)^{*}$ & $56.4(15.8)^{*}$ & $<0.001$ \\
\hline$\left[{ }^{18}\right.$ F]Florbetapir (SUVR), mean (SD) & $1(0.03)$ & $1.12(1)^{*}$ & $1.25(0.08)^{*}$ & $1.35(0.07)^{*}$ & $<0.001$ \\
\hline Follow-up (months), mean (SD) & $24.3(0.98)$ & $23.8(0.9)$ & $24.1(1.8)$ & $24(0.7)$ & 0.53 \\
\hline \multicolumn{6}{|l|}{ Diagnostic at follow-up visit, $n(\%)$} \\
\hline Cognitively normal & $22(91)$ & $55(87.5)$ & $14(88)$ & $12(71)$ & - \\
\hline Mild cognitive impairment & $2(9)$ & $7(11)$ & $2(12)$ & $5(29)$ & 0.2 \\
\hline Dementia & 0 & $1(1.5)$ & 0 & 0 & - \\
\hline
\end{tabular}

The values in each group were compared using analysis of covariance for each variable except gender, $A P O E-\varepsilon 4$ carrier status, and diagnostic at followup, for which the chi-squared test was used.

MMSE Mini-Mental State Examination

$* P<0.05$, vs. biomarkers-negative group, post-hoc analysis

enriched by including those with both biomarkers above the proposed thresholds $\left(\left[{ }^{18} \mathrm{~F}\right]\right.$ florbetapir SUVR $>1.228$ and $\mathrm{p}$-tau $>45 \mathrm{pg} / \mathrm{ml}$ ) would require 87 individuals with preclinical AD per trial arm to test a $25 \%$ drug effect ( $80 \%$ of power at a $5 \%$ level) over 2 years. On the other hand, a clinical trial using the concept of preclinical AD with standard thresholds $\left(\left[{ }^{18} \mathrm{~F}\right]\right.$ florbetapir SUVR $>1.15$ and $\mathrm{p}$-tau $>23 \mathrm{pg} / \mathrm{ml}$ ) would require 416 individuals per arm, whereas a trial without a population enrichment strategy would require 738 individuals per arm.

\section{Discussion}

In summary, our results suggest that the $A \beta$ and tau thresholds associated with imminent AD-related metabolic decline in individuals with preclinical AD are higher than their respective thresholds for abnormality. In addition, we showed that the use of these thresholds and the use of voxel-wise changes in $\left[{ }^{18} \mathrm{~F}\right] \mathrm{FDG}$ uptake as a surrogate marker as inclusion criteria provide an alternative framework with a high statistical power for 2-year clinical trials in preclinical AD. Overall, we propose the new concept of $A \beta$ and tau thresholds for imminent neurodegeneration as a valuable approach to population enrichment in clinical trials in individuals with preclinical $\mathrm{AD}$ and a high probability of developing AD-related neurodegeneration within a short time. Specifically, we demonstrated that a 2-year clinical trial in cognitively normal individuals using brain $\mathrm{A} \beta$ plus CSF p-tau thresholds more than one SD higher than their standard values together with regional $\left[{ }^{18} \mathrm{~F}\right] \mathrm{FDG}$ uptake as a surrogate marker would require as few as 100 individuals to test a hypothetical $25 \%$ drug effect. These results contrast with a recent study performed by ADNI in individuals with preclinical $\mathrm{AD}$ defined as those with $\mathrm{A} \beta$ plus tau positivity which showed that a 2-year clinical trial measuring changes in cognition or structural MRI would require more than 2,000 individuals to test a hypothetical $25 \%$ drug effect with $80 \%$ power [3].

It is important to emphasize that the thresholds proposed here are based on dynamic biomarker changes and indicate metabolic decline over short time frames, rather than the presence or absence of pathological proteinopathies, which are best assessed with post-mortem correlations [28]. It is also important to mention that in our understanding the term "biomarker abnormality" refers to the presence of brain pathology and should be defined by thresholds that are better associated with post-mortem studies. Within the biomarker abnormality spectrum, we suggest that the existence of thresholds for imminent disease progression will identify from among individuals with biomarker abnormalities those with the highest probability of progression to a given outcome. 


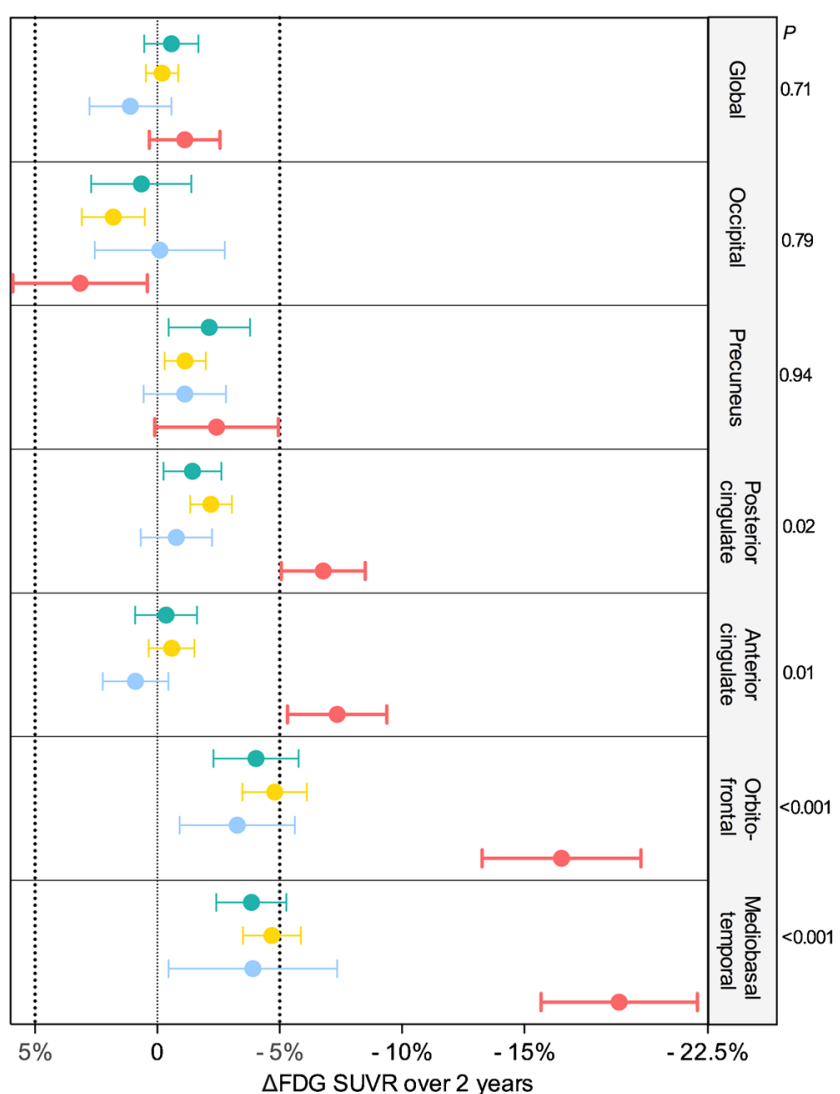

Fig. 3 In our cognitively normal population of individuals with preclinical $\mathrm{AD}$, those with $\mathrm{A} \beta$ and tau above the optimized thresholds showed the highest rates of 2-year AD-related metabolic decline. ANCOVA confirmed that these individuals (with $\left[{ }^{18} \mathrm{~F}\right]$ florbetapir SUVR $>1.23$ and $\mathrm{p}$-tau $>45 \mathrm{pg} / \mathrm{ml} ; n=17$, red) showed the highest 2year rates of $\left[{ }^{18} \mathrm{~F}\right] \mathrm{FDG}$ uptake decline in the mediobasal temporal, orbitofrontal, and cingulate cortices. It is important to emphasize that individuals with preclinical $\mathrm{AD}$ and at least one of the biomarkers below the proposed threshold $(n=16$, blue $)$ showed declines in $\left[{ }^{18} \mathrm{~F}\right] \mathrm{FDG}$ uptake not different from biomarker-negative individuals $(n=24$, green) and those at risk of $\mathrm{AD}(n=63$, yellow). The analysis was adjusted for age, gender and $A P O E-\varepsilon 4$ carrier status, with Bonferroni correction at a significance level of 0.05

Since the proposed model optimizes neurodegeneration as a function of $A \beta$ and tau levels, we may argue that this model has immediate applicability to provide a framework with a high statistical power for use with the emerging anti-tau and anti-amyloid therapies. Interestingly, the highest rates of metabolic decline shown by individuals with preclinical $\mathrm{AD}$ were found in the limbic structures within the cingulate, orbitofrontal, and medial and basal temporal cortices. Importantly, this pattern of metabolic dysfunction is not considered part of normal ageing [29, 30]. In fact, metabolic decline in the limbic structures has been proposed as an early abnormality associated with $\mathrm{AD}[31,32]$. In addition, the fact that this brain circuit is affected by tangles or plaques [29, 30], rather than by the normal ageing process [33-35], further supports the notion that cognitively normal subjects with these baseline biomarker signatures are on the AD pathway. Interestingly, in contrast with $\left[{ }^{18} \mathrm{~F}\right]$ florbetapir, $\mathrm{CSF} A \beta$ levels failed to model imminent metabolic decline in our population, which is in line with previous observations showing that $A \beta$ PET depicts metabolic decline better than CSF $A \beta$ in this preclinical AD population [13]. Although CSF A $\beta$ well-represents the disease status, brain fibrillar $A \beta$ deposition seems to be better associated with imminent disease progression $[13,36]$.

Declines in $\left[{ }^{18} \mathrm{~F}\right] \mathrm{FDG}$ uptake as a function of the hallmark $\mathrm{AD}$ proteins were best described by a sigmoidal rather than a linear association. This pattern of relationship is consistent with the most accepted models of AD progression [22], which assume "ceiling effects" for $\mathrm{A} \beta$ and tau. Therefore, our results further emphasize that this ceiling effect should be considered in studies testing the relationship between these proteinopathies and downstream neurodegeneration. For example, studies using linear functions for testing the association between proteins and brain hypometabolism in AD patients might not show any correlation [13], since it is likely that most demented patients have already reached the plateau of the relationship.

Methodological aspects limit the external validity of the present results. Our population was composed of self-selected individuals and might not represent the general elderly cognitively normal population. Therefore, it would be highly desirable to replicate our results in a larger population-based study. However, it is important to mention that the use of a conservative multiple comparison correction threshold of 0.001 helped to avoid false-positives results in our analysis. It is also important to emphasize that change in cognition is always the most desirable outcome for a therapeutic clinical trial aiming to mitigate AD progression. However, due to the methodological limitations in the use of cognition in individuals with preclinical $\mathrm{AD}$ and the advances in in vivo biomarkers, increasing our understanding of the use of brain imaging in the assessment of $\mathrm{AD}$ progression is of paramount importance. Importantly, individuals with preclinical AD in this study were considered those with normal cognition but $A \beta$ and tau abnormalities rather than those who would certainly have developed dementia over time. The 2-year follow-up was not sufficient to draw definite conclusions regarding clinical progression from cognitively normal to dementia.

Partial volume correction did not translate into differences in our final results. Therefore, we present the PET data without corrections for volume effects. Biomarker thresholds are invariably subject to idiosyncrasies and as such might vary slightly depending on the analytical method used. However, in this study, biomarker levels predictive of rapid metabolic decline were at least one SD higher than the threshold for the 


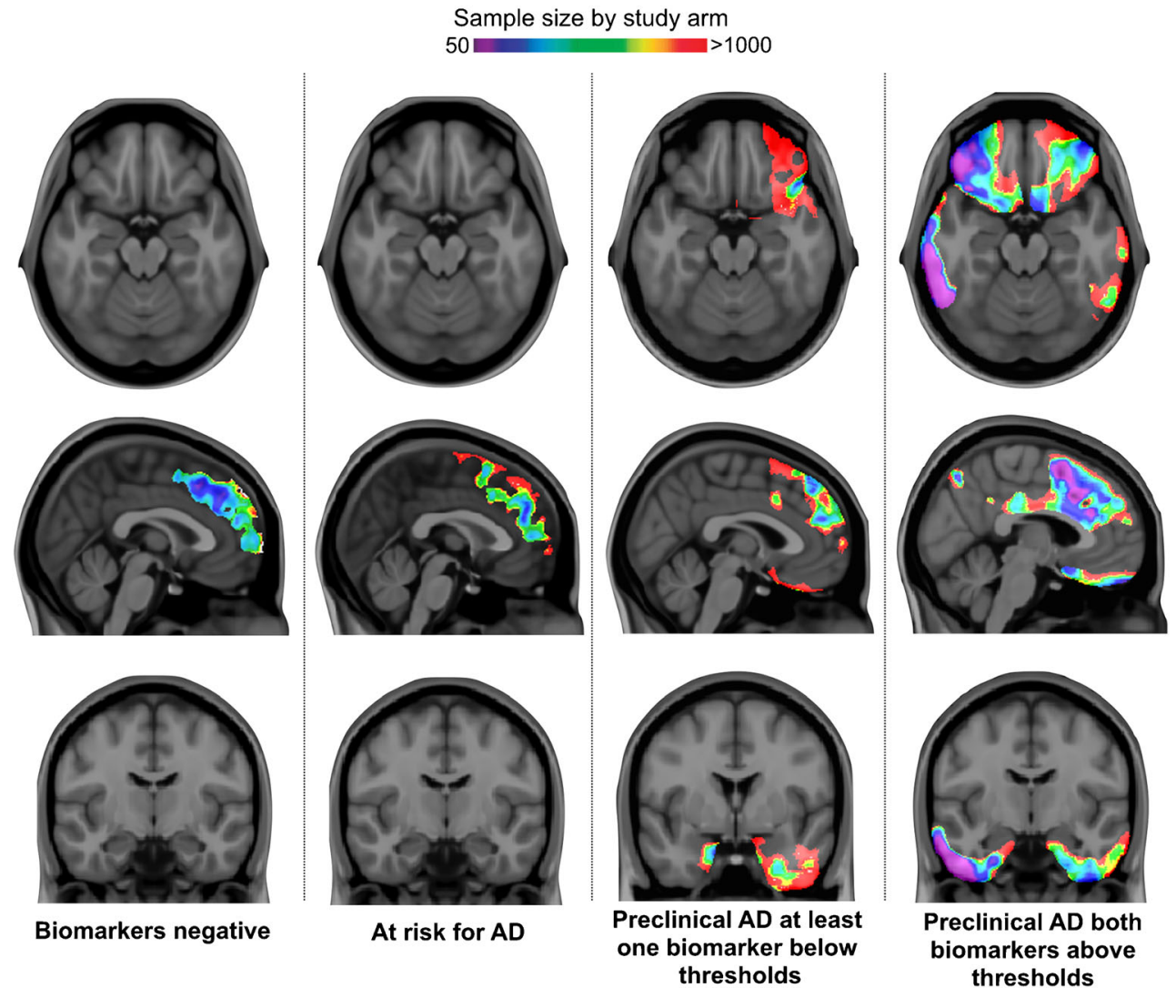

Fig. 4 Voxel-wise power analysis confirmed that defining preclinical AD according to the thresholds predictive of imminent metabolic decline offers a robust framework with a high statistical power for population enrichment of clinical trials using regional $\Delta\left[{ }^{18}\right.$ F $]$ FDG uptake as a surrogate marker. The parametric maps represent the voxel-wise sample size calculations overlain on structural MRI scans showing the regions with a significant decline in $\left[{ }^{18} \mathrm{~F}\right] \mathrm{FDG}$ uptake in each biomarker group

presence of brain pathology in an elderly cognitively normal population. Notably, the thresholds associated with imminent disease progression would invariably be expected to be higher than those used to determine an abnormal biomarker status. It is important to mention that the use of a hypothetical drug effect of $25 \%$ used in our analysis was the same as that used in previous studies in $\mathrm{AD}[3,24,25,27]$. Although study population enrichment with two biomarker modalities might potentially have a high economic cost, it is important to emphasize that an increasingly large number of observational studies using multiple biomarkers for $\mathrm{A} \beta$ and tau have indicated that this approach could serve as a screening tool to select individuals for therapeutic trials. Despite the current lack of an effective disease-modifying therapy for $\mathrm{AD}$, the promising recent results with anti-A $\beta$ drugs such as aducanumab suggest the need for sensitive methods to detect disease progression.

To conclude, our results show that $A \beta$ and tau biomarker thresholds associated with imminent neurodegeneration are

after multiple comparison correction (false discovery rate at $P<0.001$ ). The maps confirm that individuals with both biomarkers above the threshold of imminent metabolic decline provide the best framework to test a $25 \%$ drug effect on changes in $\left[{ }^{18} \mathrm{~F}\right] \mathrm{FDG}$ uptake in large clusters in the mediobasal temporal, cingulate, and orbitofrontal cortices, requiring a sample as small as 50 individuals per trial arm

higher than their respective thresholds for abnormality. In addition, the determination of these thresholds may provide complementary information for selecting individuals with preclinical AD who will most likely show pathophysiological progression over time frames compatible with clinical trials.

Acknowledgements Data collection and sharing for this project was funded by the Alzheimer's Disease Neuroimaging Initiative (ADNI; National Institutes of Health grant U01 AG024904) and DOD ADNI (Department of Defense award number W81XWH-12-2-0012). ADNI is funded by the National Institute on Aging and the National Institute of Biomedical Imaging and Bioengineering, and through generous contributions from the following: AbbVie, Alzheimer's Association, Alzheimer's Drug Discovery Foundation, Araclon Biotech, BioClinica, Biogen, Bristol-Myers Squibb Company, CereSpir, Eisai Inc., Elan Pharmaceuticals, Eli Lilly and Company, EuroImmun, F. Hoffmann-La Roche and its affiliated company Genentech, Fujirebio, GE Healthcare, IXICO, Janssen Alzheimer Immunotherapy Research \& Development, Johnson \& Johnson Pharmaceutical Research \& Development, Lumosity, Lundbeck, Merck, Meso Scale Diagnostics, NeuroRx Research, Neurotrack Technologies, Novartis Pharmaceuticals Corporation, Pfizer, Piramal Imaging, Servier, Takeda Pharmaceutical Company, and Transition Therapeutics. The Canadian Institutes of Health Research providies funds to support ADNI clinical sites in 
Canada. Private sector contributions are facilitated by the Foundation for the National Institutes of Health (www.fnih.org). The grantee organization is the Northern California Institute for Research and Education, and the study was coordinated by the Alzheimer's Disease Cooperative Study at the University of California, San Diego. ADNI data are disseminated by the Laboratory of Neuro Imaging at the University of Southern California.

Author contributions T.A.P., S.G. and P.R-N. conceived the study. T.A.P., S. Mathotaarachchi, M.S., A.L.B., S. Mohades, M.S.K. and G.M performed the processing and the quality control of the image data. T.A.P., S.M. and P.R.-N. analysed and interpreted the data. A.Y.P. assisted in the statistical analysis. T.A.P., M.S., J.-P.S., S.G., and P.R-N. prepared the figures and the table and drafted the manuscript.

Funding This work was supported by the Canadian Institutes of Health Research (CIHR; MOP-11-51-31), the Alan Tiffin Foundation, the Alzheimer's Association (NIRG-12- 92090, NIRP-12-259245), a Prevent-AD scholarship (T.A.P.), Fonds de Recherche du Québec Santé (FRQS; Chercheur Boursier, P.R.-N.), S.G. and P.R.-N. are members of the CIHR-CCNA Canadian Consortium of Neurodegeneration in Aging.

\section{Compliance with ethical standards}

Conflict of interest Serge Gauthier received honoraria for serving on the scientific advisory boards of Alzheon, Axovant, Lilly, Lundbeck, Novartis, Schwabe, TauRx, on the Data Safety Monitoring Board of a study sponsored by Eisai, and studies run by the Alzheimer's Disease Cooperative Study and by the Alzheimer's Therapeutic Research Institute. All the other authors declare no conflicts of interest.

Gassan Massarweh declares that he has no conflict of interest

Ethical approval All procedures performed in studies involving human participants were in accordance with the ethical standards of the institutional and/or national research committee and with the principles of the 1964 Declaration of Helsinki and its later amendments or comparable ethical standards.

Informed consent Informed consent was obtained from all individual participants included in the study.

Open Access This article is distributed under the terms of the Creative Commons Attribution 4.0 International License (http:// creativecommons.org/licenses/by/4.0/), which permits unrestricted use, distribution, and reproduction in any medium, provided you give appropriate credit to the original author(s) and the source, provide a link to the Creative Commons license, and indicate if changes were made.

\section{References}

1. Dubois B, Hampel H, Feldman HH, Scheltens P, Aisen P, Andrieu $\mathrm{S}$, et al. Preclinical Alzheimer's disease: definition, natural history, and diagnostic criteria. Alzheimers Dement. 2016;12:292-323. https://doi.org/10.1016/j.jalz.2016.02.002.

2. Sperling R, Mormino E, Johnson K. The evolution of preclinical Alzheimer's disease: implications for prevention trials. Neuron. 2014;84:608-22. https://doi.org/10.1016/j.neuron.2014.10.038.

3. Holland D, McEvoy LK, Desikan RS, Dale AM; Alzheimer's Disease Neuroimaging Initiative. Enrichment and stratification for predementia Alzheimer disease clinical trials. PLoS One. 2012;7: e47739. https://doi.org/10.1371/journal.pone.0047739.

4. Gauthier S, Albert M, Fox N, Goedert M, Kivipelto M, MestreFerrandiz J, et al. Why has therapy development for dementia failed in the last two decades? Alzheimers Dement. 2016;12:60-4. https:// doi.org/10.1016/j.jalz.2015.12.003.

5. Sperling RA, Aisen PS, Beckett LA, Bennett DA, Craft S, Fagan AM, et al. Toward defining the preclinical stages of Alzheimer's disease: recommendations from the National Institute on AgingAlzheimer's Association workgroups on diagnostic guidelines for Alzheimer's disease. Alzheimers Dement. 2011;7:280-92. https:// doi.org/10.1016/j.jalz.2011.03.003.

6. Fouquet M, Desgranges B, Landeau B, Duchesnay E, Mezenge F, de la Sayette V, et al. Longitudinal brain metabolic changes from amnestic mild cognitive impairment to Alzheimer's disease. Brain. 2009;132:2058-67. https://doi.org/10.1093/brain/awp132.

7. Jagust W, Reed B, Mungas D, Ellis W, Decarli C. What does fluorodeoxyglucose PET imaging add to a clinical diagnosis of dementia? Neurology. 2007;69:871-7. https://doi.org/10.1212/01. wnl.0000269790.05105.16.

8. Torosyan N, Mason K, Dahlbom M, Silverman DHS; Alzheimer's Disease Neuroimaging Initiative. Value of FDG-PET scans of nondemented patients in predicting rates of future cognitive and functional decline. Eur J Nucl Med Mol Imaging. 2017;44:1355-63. https://doi.org/10.1007/s00259-017-3634-3.

9. Morbelli S, Bauckneht M, Arnaldi D, Picco A, Pardini M, Brugnolo A, et al. 18F-FDG PET diagnostic and prognostic patterns do not overlap in Alzheimer's disease (AD) patients at the mild cognitive impairment (MCI) stage. Eur J Nucl Med Mol Imaging. 2017;44: 2073-83; https://doi.org/10.1007/s00259-017-3790-5.

10. Edison P, Archer HA, Hinz R, Hammers A, Pavese N, Tai YF, et al. Amyloid, hypometabolism, and cognition in Alzheimer disease: an [11C]PIB and [18F]FDG PET study. Neurology. 2007;68:501-8. https://doi.org/10.1212/01.wnl.0000244749.20056.d4

11. Bruck A, Virta JR, Koivunen J, Koikkalainen J, Scheinin NM, Helenius $\mathrm{H}$, et al. [11C]PIB, [18F]FDG and MR imaging in patients with mild cognitive impairment. Eur J Nucl Med Mol Imaging. 2013;40:1567-72. https://doi.org/10.1007/s00259-013-2478-8.

12. Duyckaerts $\mathrm{C}$. Tau pathology in children and young adults: can you still be unconditionally baptist? Acta Neuropathol. 2011;121:1457. https://doi.org/10.1007/s00401-010-0794-7.

13. Pascoal TA, Mathotaarachchi S, Mohades S, Benedet AL, Chung $\mathrm{CO}$, Shin M, et al. Amyloid-beta and hyperphosphorylated tau synergy drives metabolic decline in preclinical Alzheimer's disease. Mol Psychiatry. 2017;22:306-11. https://doi.org/10.1038/mp. 2016.37.

14. Pascoal TA, Mathotaarachchi S, Shin M, Benedet AL, Mohades S, Wang S, et al. Synergistic interaction between amyloid and tau predicts the progression to dementia. Alzheimers Dement. 2017;13:644-53. https://doi.org/10.1016/j.jalz.2016.11.005.

15. Toledo JB, Xie SX, Trojanowski JQ, Shaw LM. Longitudinal change in CSF Tau and $\mathrm{A} \beta$ biomarkers for up to 48 months in ADNI. Acta Neuropathol. 2013;126:659-70. https://doi.org/10. 1007/s00401-013-1151-4.

16. Shaw LM, Vanderstichele H, Knapik-Czajka M, Clark CM, Aisen PS, Petersen RC, et al. Cerebrospinal fluid biomarker signature in Alzheimer's Disease Neuroimaging Initiative subjects. Ann Neurol. 2009;65:403-13. https://doi.org/10.1002/ana.21610.

17. Eskildsen SF, Coupe P, Fonov V, Manjon JV, Leung KK, Guizard $\mathrm{N}$, et al. BEaST: brain extraction based on nonlocal segmentation technique. Neuroimage. 2012;59:2362-73. https://doi.org/10.1016/ j.neuroimage.2011.09.012.

18. Zijdenbos AP, Forghani R, Evans AC. Automatic "pipeline" analysis of 3-D MRI data for clinical trials: application to multiple sclerosis. IEEE Trans Med Imaging. 2002;21:1280-91. https:// doi.org/10.1109/TMI.2002.806283. 
19. Landau SM, Mintun MA, Joshi AD, Koeppe RA, Petersen RC, Aisen PS, et al. Amyloid deposition, hypometabolism, and longitudinal cognitive decline. Ann Neurol. 2012;72:578-86. https://doi. org/10.1002/ana.23650.

20. Joshi AD, Pontecorvo MJ, Clark CM, Carpenter AP, Jennings DL, Sadowsky $\mathrm{CH}$, et al. Performance characteristics of amyloid PET with florbetapir F18 in patients with Alzheimer's disease and cognitively normal subjects. J Nucl Med. 2012;53:378-84. https://doi. org/10.2967/jnumed.111.090340.

21. Mazziotta J, Toga A, Evans A, Fox P, Lancaster J, Zilles K, et al. A probabilistic atlas and reference system for the human brain: international consortium for brain mapping (ICBM). Philos Trans R Soc Lond B Biol Sci. 2001;356:1293-322. https://doi.org/10.1098/rstb. 2001.0915.

22. Jack CR Jr, Knopman DS, Jagust WJ, Petersen RC, Weiner MW, Aisen PS, et al. Tracking pathophysiological processes in Alzheimer's disease: an updated hypothetical model of dynamic biomarkers. Lancet Neurol. 2013;12:207-16. https://doi.org/10. 1016/S1474-4422(12)70291-0.

23. Mathotaarachchi S, Wang S, Shin M, Pascoal TA, Benedet AL, Kang MS, et al. VoxelStats: a MATLAB package for multi-modal voxel-wise brain image analysis. Front Neuroinform. 2016;10:20. https://doi.org/10.3389/fninf.2016.00020.

24. Leung KK, Clarkson MJ, Bartlett JW, Clegg S, Jack CR Jr, Weiner $\mathrm{MW}$, et al. Robust atrophy rate measurement in Alzheimer's disease using multi-site serial MRI: tissue-specific intensity normalization and parameter selection. Neuroimage. 2010;50:516-23. https:/doi. org/10.1016/j.neuroimage.2009.12.059.

25. Grill JD, Di L, Lu PH, Lee C, Ringman J, Apostolova LG, et al. Estimating sample sizes for predementia Alzheimer's trials based on the Alzheimer's disease Neuroimaging initiative. Neurobiol Aging. 2013;34:62-72. https://doi.org/10.1016/j.neurobiolaging.2012.03. 006.

26. Benjamini Y, Hochberg Y. Controlling the false discovery rate: a practical and powerful approach to multiple testing. J R Stat Soc Series B Stat Methodol. 1995;57:289-300.

27. Fox NC, Cousens S, Scahill R, Harvey RJ, Rossor MN. Using serial registered brain magnetic resonance imaging to measure disease progression in Alzheimer disease: power calculations and estimates of sample size to detect treatment effects. Arch Neurol. 2000;57: 339-44.

28. Villeneuve S, Rabinovici GD, Cohn-Sheehy BI, Madison C, Ayakta N, Ghosh PM, et al. Existing Pittsburgh compound-B positron emission tomography thresholds are too high: statistical and pathological evaluation. Brain. 2015;138:2020-33. https://oi.org/ 10.1093/brain/awv112.

29. Kuhl DE, Metter EJ, Riege WH, Hawkins RA. The effect of normal aging on patterns of local cerebral glucose utilization. Ann Neurol. 1984;15(Suppl):S133-7.

30. Moeller JR, Ishikawa T, Dhawan V, Spetsieris P, Mandel F, Alexander GE, et al. The metabolic topography of normal aging. J Cereb Blood Flow Metab. 1996;16:385-98. https://doi.org/10. 1097/00004647-199605000-00005.

31. Lowe VJ, Weigand SD, Senjem ML, Vemuri P, Jordan L, Kantarci $\mathrm{K}$, et al. Association of hypometabolism and amyloid levels in aging, normal subjects. Neurology. 2014;82:1959-67. https://doi. org/10.1212/WNL.0000000000000467.

32. Mosconi L, Mistur R, Switalski R, Brys M, Glodzik L, Rich K, et al. Declining brain glucose metabolism in normal individuals with a maternal history of Alzheimer disease. Neurology. 2009;72:513-20. https://doi.org/10.1212/01.wnl.0000333247. 51383.43 .

33. Thal DR, Rub U, Orantes M, Braak H. Phases of a beta-deposition in the human brain and its relevance for the development of AD. Neurology. 2002;58:1791-800.

34. Delacourte A, David JP, Sergeant N, Buee L, Wattez A, Vermersch $\mathrm{P}$, et al. The biochemical pathway of neurofibrillary degeneration in aging and Alzheimer's disease. Neurology. 1999;52:1158-65.

35. Braak H, Braak E. Neuropathological stageing of Alzheimerrelated changes. Acta Neuropathol. 1991;82:239-59.

36. Mattsson N, Insel PS, Donohue M, Landau S, Jagust WJ, Shaw $\mathrm{LM}$, et al. Independent information from cerebrospinal fluid amyloid-beta and florbetapir imaging in Alzheimer's disease. Brain. 2015;138:772-83. https://doi.org/10.1093/brain/awu367. 